\title{
Subway Station Capacity Maintained by Optimizing a Maintenance Schedule of Key Equipment
}

\author{
Sai Li ${ }^{1,2}$, Xiukun Wei ${ }^{1, *(\mathbb{D})}$, Zhe Zhang ${ }^{1}$, Limin Jia ${ }^{1}$ and Junyan $\mathrm{Yu}^{3}$ \\ 1 State Key Laboratory of Rail Traffic Control and Safety, Beijing Jiaotong University, Beijing 100044, China; \\ 17120965@bjtu.edu.cn (S.L.); zhangzhe@bjtu.edu.cn (Z.Z.); lmjia@bjtu.edu.cn (L.J.) \\ 2 School of Traffic and Transportation, Beijing Jiaotong University, Beijing 100044, China \\ 3 Guangzhou Metro Group Co. Ltd., Guangzhou 510330, China; yujunyan@gzmtr.com \\ * Correspondence: xkwei@bjtu.edu.cn
}

Received: 23 February 2019; Accepted: 26 March 2019; Published: 2 April 2019

\begin{abstract}
The service capacity of each station has a direct impact on the capacity of a subway line and also the whole subway network. Therefore, it is critically important to keep the station capacity above a certain level in its full life cycle. This paper aims at keeping subway station capacity in a certain level range. In consideration of key equipment failure, the expected station capacity model is established. After that the lower bound of equipment reliability is determined by sensitivity analysis based on the equipment reliability-expected station capacity curve. Finally, the maintenance schedule optimization model is also proposed. The validity and practicability of the proposed method are demonstrated by a simulation case study.
\end{abstract}

Keywords: subway station capacity; key equipment; sensitivity analysis; reliability; maintenance schedule

\section{Introduction}

Subway stations play an important role in urban rail transit networks since passengers gather for boarding or alight at these locations. The service capacity of each station has a direct impact on the capacity of subway line and also on the whole subway network. Therefore, it is critically important to keep the station service capacity above an acceptable level in its full life cycle. The station equipment's performance deteriorates and its reliability decreases over time. When station equipment fails, station capacity declines and the travel time of passengers increases, especially during rush hour periods. Therefore, how to optimize key equipment maintenance schedule to maintain the station capacity in the full life cycle is significantly important for subway operation companies.

The subway station capacity (SSC) issue has received some attention in the last two decades (see references [1-15]). There are a few definitions involving SSC in the available literature. In references [1,2], SSC is defined as the maximum passenger flow through various facilities, such as stairs, escalators and gates, at one subway station. However, the influence of passenger flow is not considered in this definition. To solve the problem considered in this paper, dynamic SSC is defined later according to the gathering and scattering process of passengers. There have been a few methods to calculate or evaluate SSC in the available literature. The methods are classified into two main categories according to the modeling approach: the mathematical model-based method and the simulation-based method. The first approach evaluates the subway station system by means of mathematical formulas. The $\mathrm{M} / \mathrm{G} / \mathrm{C} / \mathrm{C}$ state dependent queuing network is used in mathematical models of subway stations, especially for the staircase and the pavement (see references [3-6]). In reference [7], an M/G/C/C-based capacity model of staircases and corridors is proposed for passenger evacuation in subway stations through analysis of passenger movements. However, there 
is little research on the evaluation or that calculates SSC in a holistic fashion. In references [8,9], an analytical queuing network model for a subway station including two subsystems is built, of which the first subsystem is built by the M/G/C/C state dependent queuing network and the second is constructed by the probability theory. However, equipment failure is not considered in the station capacity model which affects SSC. Simulation models (see references [10-15]) are suitable for animating stations in different perspectives, but only a few researchers have considered the capacity calculation problem. For example, a simulation model based on hybrid Petri nets is presented in [11]. A modeling approach based on discrete-event simulation and response surface methodology (RSM) deals with the optimization of average passenger travel time inherent in the metro planning process in reference [12]. In the references [13,14], the station simulation model based on AnyLogic is established to simulate the gathering and scattering process and evaluate the usage of device. In reference [15], an AnyLogic model is established to analyze the current status of the subway station. These studies focused on how to model the station, evaluate the usage of device or identify bottlenecks of a station. However, a method to calculate the station capacity integrating simulation models has not been reported yet. The passenger carrying capacity of a rail transit station is calculated by simulation model based on the system dynamics theory [2]. Similarly, the software AnyLogic based on social force model can be used to calculate SSC.

The reliability analysis of the key equipment such as the gate and the escalator play a critical role in our study. However, little research on reliability analysis of key equipment at subway stations has been conducted, to the best of our knowledge. The only research achievement has been to classify the common equipment faults and find out the fault causes. Gates and escalators belong to electromechanical equipment, and the methods to analyze their reliability are divided into the statistical model and the mechanism model. In this paper, the statistical model is used to analyze the reliability of equipment. Since there is no literature focusing on the reliability of station equipment, the idea of the reliability research on high-speed train bogie system is employed to analyze the reliability of key station equipment. In references [16-18], the fault data of the bogie system is collected and the reliability of the bogie is analyzed based on the statistical method. In reference [19], each component of the bogie system is estimated based on maximum likelihood estimation and the optimal distribution is determined by the A-D test method. There are many other works on the reliability analysis of mechanical equipment. Not all of the references used are listed here.

With regard to the maintenance schedule optimization, the methods of the preventive maintenance schedule model are systematically introduced and the reliability constraints are added to the preventive maintenance schedule model in reference [20]. In reference [21], life distribution of key train operation equipment is determined by the statistical method, and the optimization model of maintenance schedule is established. In reference [22], the operation and maintenance status transition diagram of the whole service life of the equipment is given, and the relationship model between the maintenance schedule and the average availability of the equipment during the whole service life is established. Based on reliability analysis of the key components of rail transit vehicles, the optimization model of the vehicle maintenance schedule is established with the minimum maintenance cost as the goal in reference [23]. This research provided useful maintenance schedule optimization models, but these models take the entire equipment as the research object which lacks pertinence, especially for electromechanical equipment since the failure rate of each component varies greatly. Therefore, it is necessary to build a maintenance schedule optimization model for the components of electromechanical equipment.

This paper aims at keeping or even enhancing SSC in the full life cycle. To investigate this issue, the dynamic SSC (DSSC) is defined and the method to calculate the expected DSSC (also referred to as expected station capacity) with equipment failure is proposed in the first stage. The average travel time of passengers is considered as the evaluation indicator of DSSC, which is calculated by passenger simulation model based on AnyLogic (see references [13-15]). The DSSC model with key equipment failure is established based on binomial distribution and mathematical expectation. In the second stage, 
the reliability of key equipment at a subway station is investigated, and the lower bound of equipment reliability is determined by sensitivity analysis based on the equipment reliability-expected station capacity curve. In addition, the maximum likelihood estimation method (MLE) is adopted to estimate fault distribution parameters of key equipment, and the Anderson-Darling statistic is used to select the optimal distribution. Finally, an optimization method for the key equipment maintenance schedule is presented in the third stage, which aims at ensuring the reliable operation of equipment with the lowest maintenance cost so that the subway station capacity can be retained in the full life cycle.

The main contributions of the paper are as follows:

(1) The interaction between equipment failures and DSSC is analyzed, and the uncertain equipment failure model is incorporated into the DSSC model.

(2) Based on the sensitive analysis between equipment reliability and DSSC, a maintenance schedule optimization model of equipment which minimizes the maintenance cost is proposed.

This paper is organized as follows. The definition of SSC and the problem considered are introduced in Section 2. The DSSC model considering equipment failure is proposed in Section 3. The method to determine the optimal fault distribution of key equipment is presented in Section 4 . The maintenance schedule optimization of key equipment is presented in Section 5. In Section 6, how the proposed approach is applied to maintain station capacity is shown by a case study in detail. Finally, some conclusions are given in Section 7.

\section{Dynamical SSC and Problem Statement}

\subsection{Definition of Dynamical SSC}

For a typical subway station, the gathering and scattering process usually consists of three sub-procedures: arrival, departure and the alighting-boarding process, as shown in Figure 1. The arrival process begins when passengers buy tickets and ends when passengers arrive at the platform. In this process, passengers can buy tickets from the TVM (Ticket Vending Machine) or use e-tickets with their ID-Card or Smart phone. The passengers pass through the security check system and gates in the Hall, arrive at the platform by taking escalators or staircases, and board the trains. The departure process starts when passengers get off from the train, then take the escalators or staircases to the Hall and finally arrive at the leaving gates. The alighting-boarding process is a bridge connecting the arrival and departure processes.

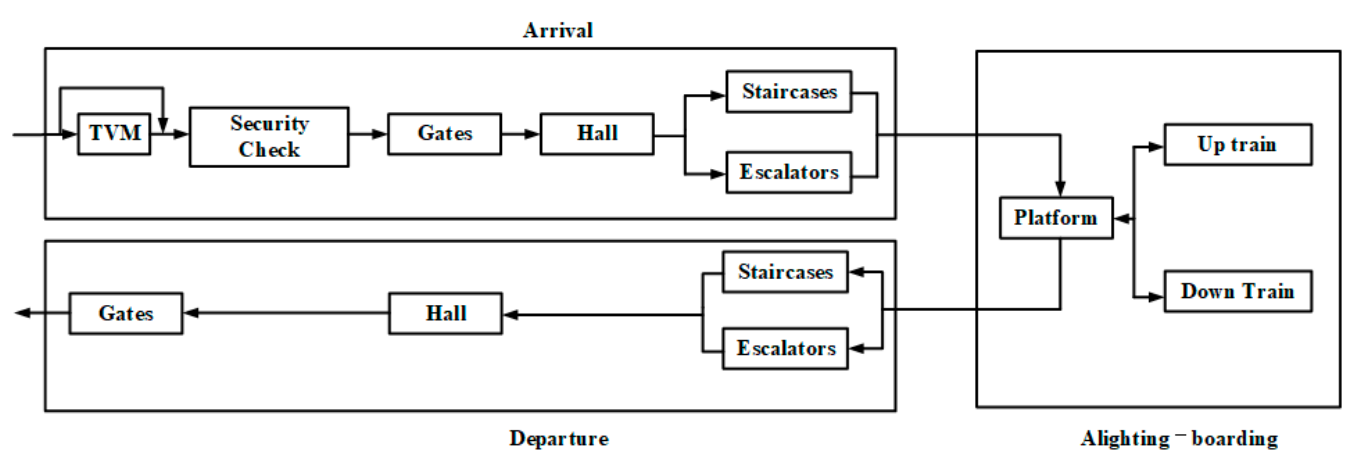

Figure 1. Passenger gathering and scattering process at a subway station.

In the gathering and scattering process, the subway station is regarded as a service system according to the queuing theory [4], as shown in Figure 2. The input is the number of passengers entering the station system per unit time, the output is the number of passengers leaving the station system per unit time. The service time includes the arrival time, departure time, and waiting time on the platform. The service time reflects the efficiency of the subway station.

The SSC is generally defined as the maximum number of passengers served by one station in a given period of time [9]. The SSC can be measured by the sum of inbound and alighting passengers or 
the sum of outbound and boarding passengers under certain level of service. It is a constant value which is related to the uncertain equipment state. In our case, the dynamical SSC (DSSC) needs to be considered. The influence of passenger inflow and the key equipment reliability on the SSC are considered. Therefore, in this paper, the DSSC is defined as the number of passengers served by one station in a given period of time, typically $1 \mathrm{~h}$, with certain passenger inflow input, under specified operating conditions [9].

\begin{tabular}{|c|c|c|c|c|}
\hline \multirow{2}{*}{$\begin{array}{l}\text { Inbound passenger } \\
\text { Alighting passenger }\end{array}$} & Input & \multirow{2}{*}{$\begin{array}{l}\text { Subway } \\
\text { station }\end{array}$} & Output & Outbound passenger \\
\hline & & & & Boarding passenger \\
\hline
\end{tabular}

Figure 2. Service system at the station.

\subsection{Evaluation Indicator of DSSC}

To evaluate the DSCC of a subway station, the metric of DSSC with certain passenger inflow needs to be defined first. Since the number of passengers served by the station per unit time only reflects the gathering and scattering efficiency from the perspective of managers, this paper uses the average travel time (ATT) of passengers at the station as the metric of DSSC. The ATT of passengers includes the travel time of arrival process and departure process. The travel time of arrival process is defined as the walking time from entrance to platform, and the travel time of departure process is defined as the walking time from platform screen door to the outside of the gates. The average travel time of passengers can be calculated as follows:

$$
T_{A}=\frac{\sum_{i=1}^{m} t_{i}^{i n}}{m}+\frac{\sum_{j=1}^{n} t_{j}^{\text {out }}}{n}
$$

where $T_{A}$ denotes the ATT of passengers, $m$ denotes the total number of people who complete the whole arrival process, $t_{i}^{i n}$ is the travel time of the arrival process, $n$ denotes the total number of people who complete the whole departure process, and $t_{j}^{\text {out }}$ is the travel time of the departure process.

\subsection{Problem Statement}

The equipment in a subway station may stop service due to the electrical and mechanical faults. The DSSC decreases in the case of equipment failure and then the travel time of passengers increases, especially during rush hours. The problem concerned in this paper is how to optimize the maintenance schedule of key equipment to keep or even enhance DSSC with the lowest maintenance cost in its full life cycle. The issue addressed is divided into three problems. One is how to build the DSSC model considering key equipment failure. The second is how to analyze the reliability of key equipment and build the fault distribution model. The final problem is how to optimize the maintenance schedule of key equipment.

As an aid to the reader, the full names of abbreviations are shown in Table 1.

Table 1. Abbreviations.

\begin{tabular}{cc}
\hline Term & Abbreviation \\
\hline Subway station capacity & SSC \\
Response surface methodology & RSM \\
Maximum likelihood estimation method & MLE \\
Ticket Vending Machine & TVM \\
Dynamical subway station capacity & DSSC \\
Average travel time & ATT \\
Inbound gate subsystem & IGS \\
Outbound gate subsystem & OGS \\
Upward escalator subsystem & UGS \\
Downward escalator subsystem & DGS \\
\hline
\end{tabular}




\section{DSSC Model with Equipment Failure}

\subsection{Key Equipment of Subway Stations}

The station facilities at a subway station are mainly divided into two types: civil engineering facilities and electromechanical equipment. The civil engineering facilities include staircases, pavements, a hall, platform, etc. The electromechanical equipment includes TVMs, gates, escalators, and platform screen doors. The reliability of civil engineering facilities is equal to almost 1 since they are not easy to damage for a long period of time after they are built. The reliability of the electromechanical equipment, such as gates and escalators, decreases along with time. The reliability of these equipment has a significant impact on the DSSC. Since the entire screen door failure hardly occurs, and one single screen door failure hardly affects the DSSC, the impact of the screen door failure on DSSC is not considered in this study. In addition, TVMs are not considered as key equipment since more and more passengers are using e-Tickets and the ratio of these passengers to the whole passengers are quite large. Therefore, gates and escalators are regarded as the key equipment which affects DSSC.

\subsection{Key Equipment Capacity Model with Equipment Failure}

The key equipment has two operation states: normal and failure. Assume that the reliability of key equipment such as the gate is $R_{e}(t)$, the maximum capacity of a single key equipment is $C_{e}$, and the capacity of key equipment is 0 when the equipment fails. At time $t$, the expected capacity of a single equipment is $C_{e} * R_{e}(t)$. In a subway station, a collection of several same types of equipment is usually used. Assume that the number of equipment in the subsystem is $n$ and the operating state of the equipment in the subsystem is independent of each other. When the number of normal equipment of the subsystem is $k$, the maximum capacity of the subsystem $C_{k}$ is $k * C_{e}$, and the probability $P_{k}$ of the operating state is $C_{n}^{k} *\left(1-R_{e}(t)\right)^{n-k} *\left(R_{e}(t)\right)^{k}$. The detailed capacity parameters is shown in Table 2 according to the binomial distribution. Therefore, the expected capacity of the subsystem with equipment failure is $\sum_{k=0}^{n}\left(k \cdot C_{e} \cdot P_{i}\right)$.

Table 2. Capacity parameters of the subsystem.

\begin{tabular}{ccccc}
\hline The Number of Normal Equipment $/ \boldsymbol{k}$ & $\mathbf{0}$ & $\mathbf{1}$ & $\ldots$ & $\boldsymbol{n}$ \\
\hline Probability $/ P_{k}$ & $\left(1-R_{e}(t)\right)^{n}$ & $C_{n}^{1} *\left(1-R_{e}(t)\right)^{n-1} * R_{e}(t)$ & $\ldots$ & $\left(R_{e}(t)\right)^{n}$ \\
The max capacity of the subsystem $/ C_{k}$ & 0 & $C_{e}$ & $\ldots$ & $n * C_{e}$ \\
\hline
\end{tabular}

\subsection{Expected DSSC Model}

When the key equipment fails, the number passengers served per unit time decreases, and DSSC also declines. In terms of the different functions of equipment at a subway station, there are four types of subsystems: the inbound gate subsystem (IGS), the outbound gate subsystem (OGS), the upward escalator subsystem (UGS) and the downward escalator subsystem (DGS), respectively, regardless of the equipment layout.

Assume that the reliability of the gate and the escalator is $R_{1}(t)$ and $R_{2}(t)$, respectively, and the number of the inbound gates, the outbound gates, the upward escalators, the downward escalators is $m_{1}, m_{2}, m_{3}$ and $m_{4}$, respectively. The number of normal gates of the inbound gate subsystem is $g$, $g=0,1,2, \ldots, m_{1}$. Therefore, there are $m_{1}+1$ operating states for IGS, and the probability of each operating state is $P_{1 g}$. Similarly, the numbers of operating states of OGS, UGS and DGS are $m_{2}+1$, $m_{3}+1$ and $m_{4}+1$, respectively, and the corresponding probability are $P_{2 h}, P_{3 d}$ and $P_{4 q}$, respectively. The values of $g, h, d$ and $q$ are independent of each other, and the number of key equipment working normally at the station is shown in Figure 3. 

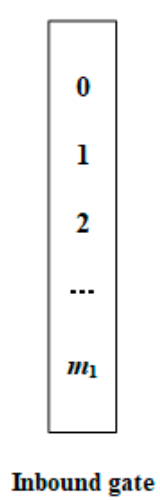
subsystem
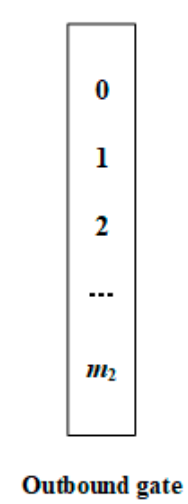
subsystem

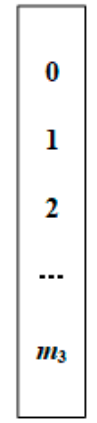

Upward escalator subsystem

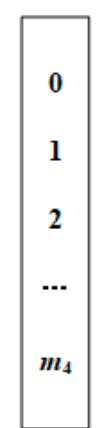

Downward escalator
subsystem

Figure 3. The number of key equipment working normally at the station.

There are $m$ operation states for subway stations and the corresponding probability and DSSC for each operation state is $P_{i}$ and $C_{i}$, respectively. To estimate the DSSC of the station, the corresponding capacity indicator such as ATT is calculated by simulation experiments with AnyLogic and Equation (1) [13]. Assuming that the passengers served by the equipment is shared equally by other facilities which play the same role at the station after it fails. For example, when an upward escalator fails, the passengers served by this escalator previously are shared equally by the other upward escalators and stairs.

The expected DSSC $C_{S}$ is defined as the expected value of DSSC considering equipment failure, which is the sum of the product of the probability of each station operation state and the corresponding DSSC. The expected travel time $T_{S}$ is an evaluation indicator of the expected DSSC, which is the expected value of AAT corresponding to each operation state of station. $C_{S}$ and $T_{S}$ are formulated as follows:

$$
\begin{gathered}
\mathrm{m}=\prod_{j=1}^{4}\left(m_{j}+1\right) \\
P_{i}=P_{1 g} \cdot P_{2 h} \cdot P_{3 d} \cdot P_{4 q} \\
C_{S}=\sum_{i=1}^{n}\left(C_{i} \cdot P_{i}\right) \\
T_{S}=\sum_{i=1}^{n}\left(T_{i} \cdot P_{i}\right)
\end{gathered}
$$

where $C_{S}$ is the expected dynamical capacity of subway station, $T_{S}$ is the expected travel time, $n$ is the total number of operation states of subway stations, $C_{i}$ is the DSSC corresponding to each operation state, $T_{i}$ is the ATT corresponding to each operation state of station which is an indicator of $C_{i}, P_{i}$ is the probability corresponding to each operation state of the station.

\section{Reliability Analysis of the Key Equipment}

\subsection{Sensitivity Analysis}

Sensitivity analysis is a method to study and analyze the sensitivity of the state or output of a system to system parameters (see reference $[9,24]$ ). By means of sensitivity analysis, the impact of equipment failure on DSSC is analyzed, and the impact of equipment reliability on the expected DSSC (also referred to as expected station capacity) is analyzed. 
Sensitivity $\phi_{i}$ represents the influence degree of the number of failed equipment on the DSSC, $\phi_{i}$ can be formulated as follows:

$$
\begin{gathered}
\phi_{i}=\frac{C_{0}-C_{i}}{C_{0}} \\
\phi_{i} \geq \beta
\end{gathered}
$$

where $i$ represents the number of failed equipment, $\phi_{i}$ is the sensitivity when the number of failed equipment is $i, C_{i}$ is the dynamical station capacity when the number of failed equipment is $n, C_{0}$ is the station capacity when no equipment fails, $\beta$ is the sensitivity threshold.

In order to keep the DSSC at a certain level, the lower bound of equipment reliability needs to be determined by sensitivity analysis based on the curve of the equipment reliability-expected station capacity.

Sensitivity $\phi_{r}$ represents the influence degree of the equipment reliability on the expected DSSC, $\phi_{r}$ can be formulated as follows:

$$
\begin{gathered}
\phi_{r}=\frac{C_{1}-C_{r}}{C_{1}} \\
\phi_{r} \geq \gamma
\end{gathered}
$$

where $r$ represents the equipment reliability, $\phi_{r}$ is the sensitivity when the equipment reliability is $r, C_{r}$ is the expected DSSC when the equipment reliability is $r, C_{1}$ is the expected DSSC when the equipment reliability is $1, \gamma$ is the sensitivity threshold.

\subsection{Reliability Modeling of the Key Equipment}

Large amounts of data on the key equipment maintenance record is available from the subway operation company. The faults of the key equipment are analyzed and divided into several classes. For each class, the probability of the failure intervals can be approximated by a typical distribution function. The commonly used fault distribution models are exponential distribution, normal distribution and Weibull distribution [19]. In this paper, the maximum likelihood estimation method (MLE) is adopted to estimate parameters of fault distributions. The likelihood function indicates how likely the observed sample is as a function of possible parameter values. Therefore, maximizing the likelihood function determines the parameters that are most likely to produce the observed data.

The Anderson-Darling statistic measures how well the data follow a particular distribution. For a particular data set and distribution, the better the distribution fits the data, the smaller this statistic will be. The Anderson-Darling statistic can be used to determine the optimal distribution of the component [19].

The Anderson-Darling statistic can be formulated as follows:

$$
\begin{gathered}
A^{2}=-n-\frac{1}{n} \sum_{i=1}^{n}(2 i-1)\left[\ln F\left(x_{i}\right)+\ln \left(1-F\left(x_{n+1-i}\right)\right)\right] \\
F\left(x_{i}\right)=\Phi\left(\frac{x_{i}-\bar{x}}{\sigma}\right)
\end{gathered}
$$

where $n$ is the sample size, $x_{i}$ is the value of the $i$ th sample, and $F\left(x_{i}\right)$ is an empirical cumulative distribution function subject to standard normal distribution.

\section{Maintenance Schedule Optimization}

The key equipment maintenance schedule optimization of subway station aims at keeping the reliable operation of equipment in a certain range with the lowest maintenance cost. For the key equipment, the failure rates of different components of the equipment are different. In order to repair the equipment more pertinently, improve the maintenance efficiency and reduce the maintenance cost, the equipment can be divided into $\mathrm{n}$ parts, and different maintenance schedules are adopted 
respectively. The maintenance schedule of the $i$ th part is $T_{i}$, the corresponding reliability function is $R_{i}(t)$, and the corresponding maintenance cost is $C_{i}\left(T_{i}\right), i \in\{0,1,2, \ldots, n\}$. Therefore, the total maintenance cost of equipment is $C_{e}\left(T_{1}, T_{2}, \cdots, T_{n}\right)$.

Therefore, with the constraint of equipment reliability, the average maintenance cost per unit maintenance schedule is taken as the optimization objective, and the maintenance schedule optimization model is established. In consideration of the impact of equipment failure on the normal operation of the station, the maintenance cost not only refers to the cost of equipment maintenance and labor, but also includes the loss of equipment downtime [21]. In addition, the maintenance cost data can be obtained from the maintenance account records. The maintenance schedule optimization model can be formulated as follows:

$$
\begin{gathered}
\min \left(C_{e}\left(T_{1}, T_{2}, \cdots, T_{n}\right)\right)=\min \left(C_{1}\left(T_{1}\right)+C_{2}\left(T_{2}\right)+\cdots C_{n}\left(T_{n}\right)\right) \\
C\left(T_{\mathrm{i}}\right)=\frac{c_{p i} R_{i}\left(T_{\mathrm{i}}\right)+c_{f i} \int_{0}^{T_{i}} f_{i}(t) d t}{\int_{0}^{T_{i}} R_{i}(t) d t} \\
R_{1}\left(T_{1}\right) * R_{2}\left(T_{2}\right) * \cdots * R_{n}\left(T_{n}\right) \geq R_{e} \\
T_{1}, T_{2}, \ldots T_{n} \in N^{*}
\end{gathered}
$$

where $C\left(T_{i}\right)$ is the expected cost per unit time of normal operation of $i$ th part of equipment within a single maintenance schedule, $c_{p_{i}}$ is the average total cost of $i$ th part of equipment within a single periodic maintenance including preventive replacement cost for non-repairable components and repair maintenance cost for repairable components, $c_{f_{i}}$ is the average total cost of $i$ th part of equipment failure including replacement cost, repair maintenance cost and the loss of equipment downtime, $R_{i}(t)$ is the reliability function of $i$ th part of the equipment, $f_{i}(t)$ is the fault probability density function of $i$ th part of the equipment, $T_{i}$ is maintenance schedule of $i$ th part which is the decision variable, $\int_{0}^{T} R(t) d t$ is the expected time of normal operation of equipment with a single maintenance schedule, $R_{e}$ is lower bound of equipment reliability which is determined by sensitivity analysis based on the equipment reliability-expected station capacity curve.

The enumeration method is adopted to solve the model, and the algorithm flow chart is shown in Figure 4. Firstly, the reliability function $R_{i}(t)$ and the fault probability density function $f_{i}(t)$ of each part of the equipment and the lower bound of equipment reliability $R_{e}$ are given before the optimization. Secondly, the minimum value of maintenance schedule $T_{i}$ for each part of the equipment which cannot meet the equipment reliability requirement are determined by loop statement, since the reliability of the equipment components decreases as service time goes by. Then the preliminary maintenance schedule collections $T_{c}$ for all of the equipment are obtained by combining the available maintenance schedules of the equipment components. Thirdly, the final maintenance schedule collections $T_{S}$ which meet the equipment reliability requirement for the entire equipment is selected from $T_{c}$. Finally, for each maintenance schedule collection of $T_{s}$, the total cost of the equipment $C_{e}$ within a single maintenance schedule is calculated. Afterwards, the minimum value is selected, and the optimal maintenance schedule for each part of the equipment is obtained. 


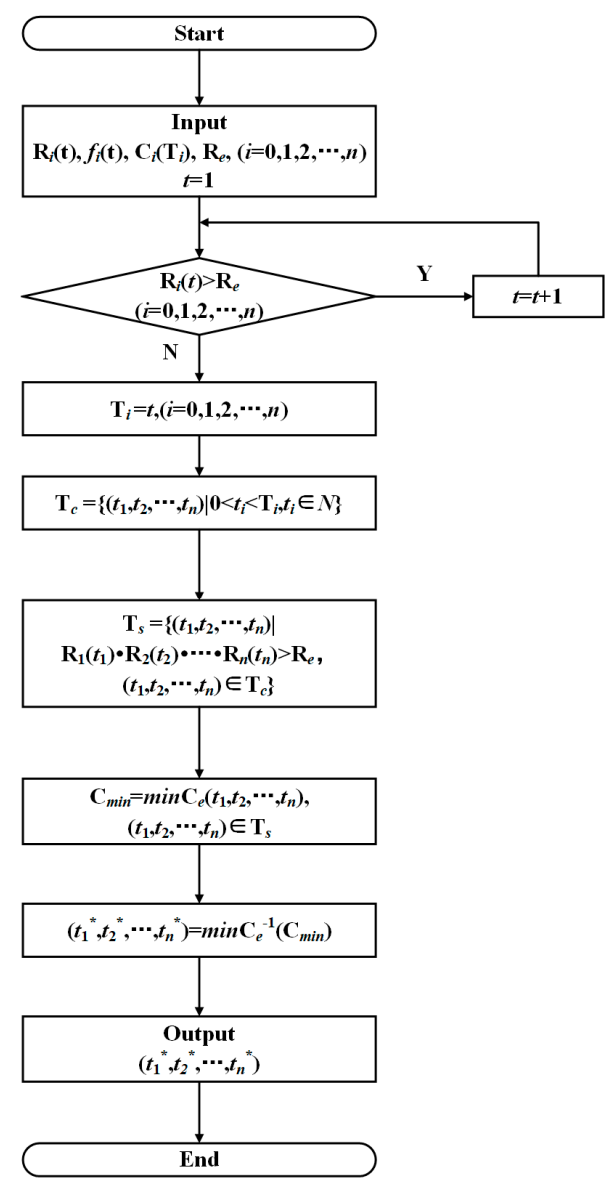

Figure 4. The flow chart for maintenance schedule optimization algorithm.

In summary, the method consists of the following steps:

(1) Passenger flow simulation model is built to calculate the ATT of the station with certain passenger inflow. For practical application, this step can be omitted.

(2) By using the sensitive analysis between equipment reliability and the expected DSSC, the boundary of equipment reliability is determined.

(3) Based on the maintenance records, gates and escalators are divided into several typical parts. The optimal fault distribution for each parts of equipment is selected from the common fault distributions with the A-D test.

(4) Finally, the related parameters of maintenance schedule optimization model are determined such as the lower bound of equipment reliability and the optimal fault distribution for each parts of equipment. The optimal maintenance schedule for key equipment is obtained by solving the model.

\section{Simulation Study}

In the former sections, the theory of the key equipment maintenance schedule optimization is presented. In this section, the proposed method will be validated by a detailed case study of a station at the Beijing subway with the help of simulation software AnyLogic.

\subsection{Passenger Flow Simulation Model}

The Xinjiekou station in Beijing subway line 4 is taken as a case study in this paper. Based on a field investigation of the station, the layout map and passenger flow data of the station are obtained. 
The length of the station hall is $60 \mathrm{~m}$, and the width is $17 \mathrm{~m}$. The length of platform is $118 \mathrm{~m}$, and the width is $9 \mathrm{~m}$. The streamline layout of the station is shown in Figure 5 .
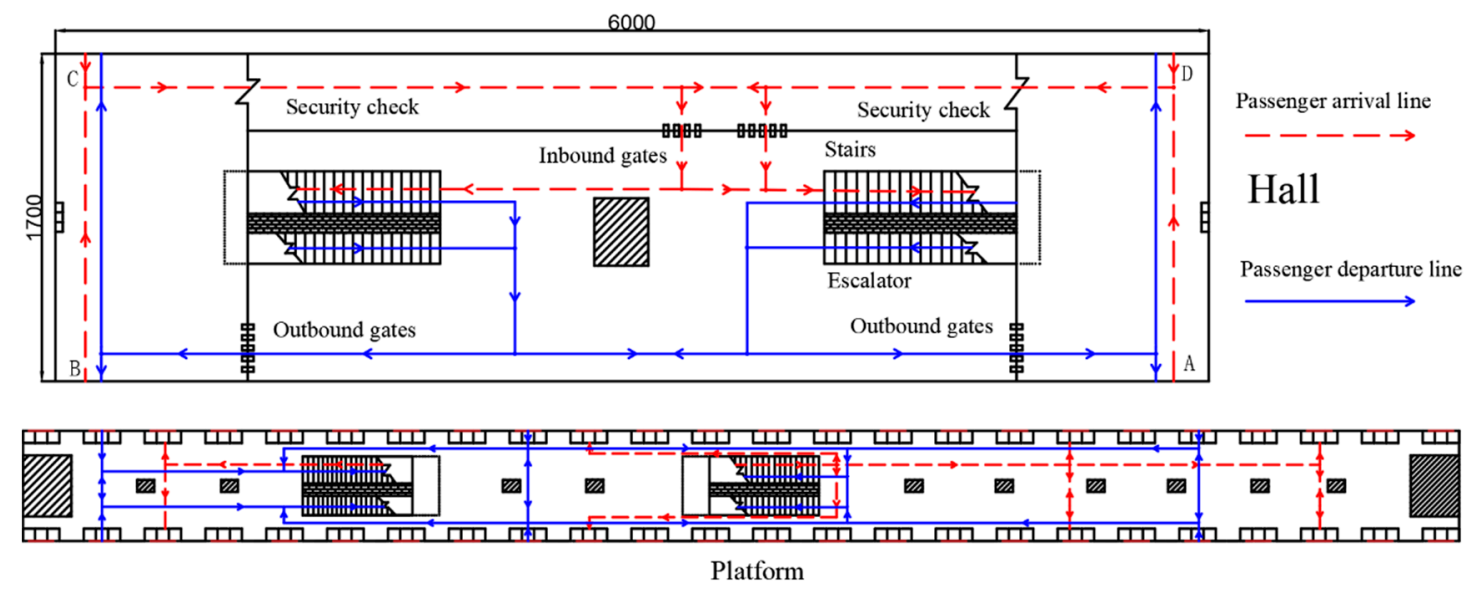

Figure 5. The streamline layout of the subway station.

The AnyLogic model consists of a station environment model and a pedestrian behavior model. In the light of the layout of station, the station environment model is established in the first place. Firstly, the buildings of the station are built including the hall and the platform, such as walls, columns and obstacles. After that, various service facilities are built in the model, such as gates and escalators, and the corresponding parameters of 'line service' and 'escalator group' are set at the same time. Finally, the pedestrian sources and destinations are set with the 'target line'. The station environment model is completed.

In the light of the layout of station, the station environment model is established, and the related environment model parameters are shown in Table 3. According to the streamline layout, the logical modules in the pedestrian library are used to construct the whole process from pedestrian generation, and the receiving services are established from various service facilities to the disappearance of pedestrians. The pedestrian behavior model is established and shown in Figures 6 and 7, respectively. It is necessary to calibrate the service parameters of the 'Ped Service' modules, and the related service parameters are shown in Table 4.

Table 3. Environment model parameters of the station.

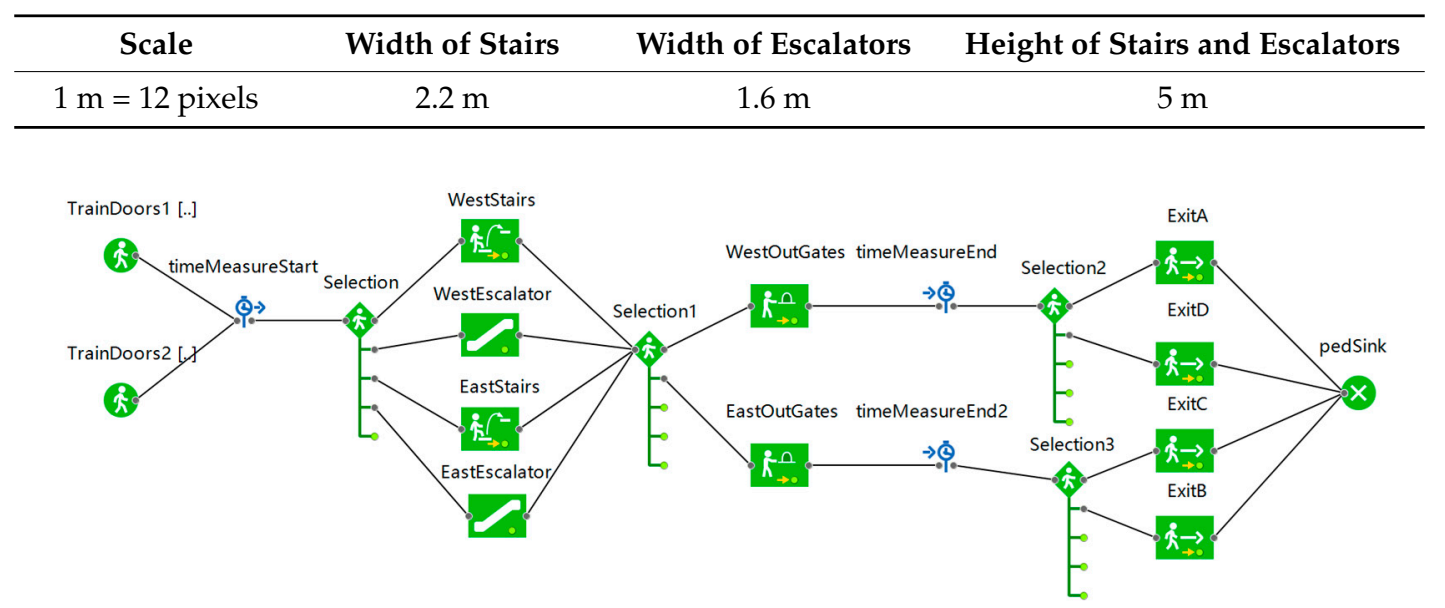

Figure 6. The outbound pedestrian behavior model. 


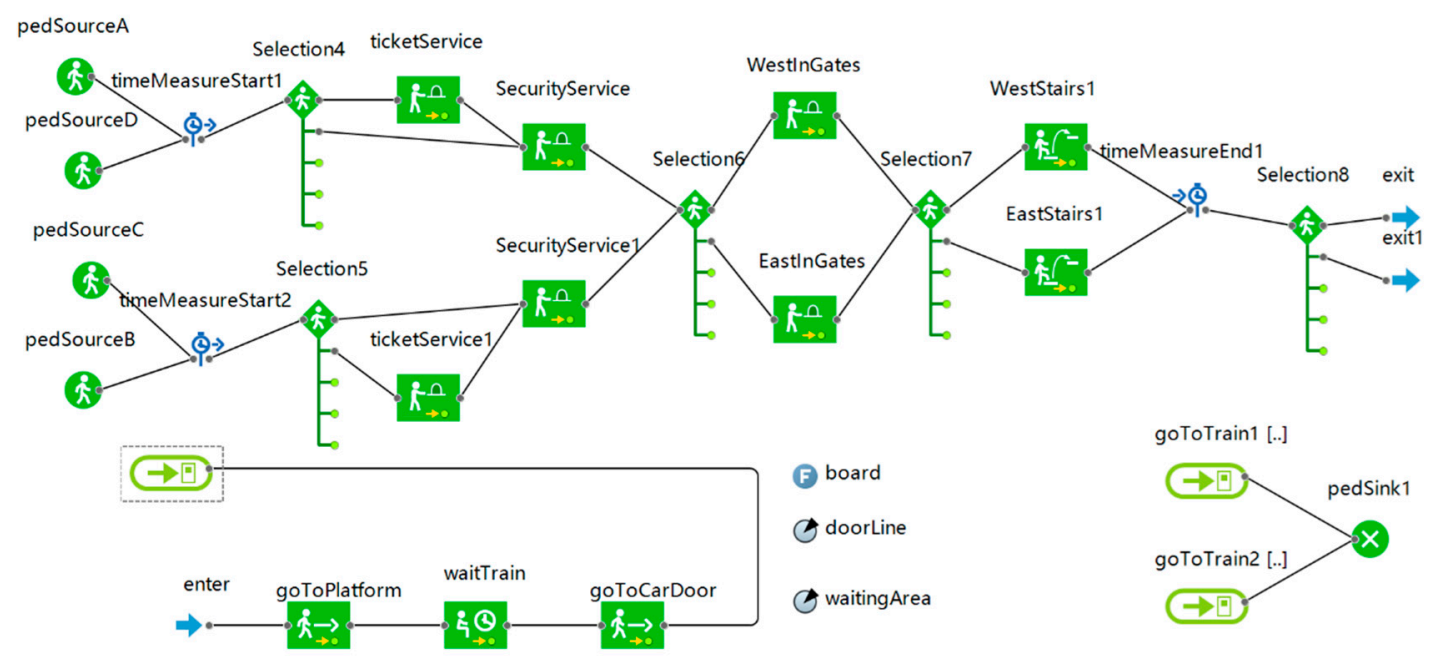

Figure 7. The inbound pedestrian behavior model.

Table 4. Service parameters of the station facilities.

\begin{tabular}{cccc}
\hline Module & Station Facilities & Parameter Name & Parameter Assignment \\
\hline \multirow{3}{*}{ Ped Service } & TVM & Delay time & normal $(12.0,40.0)^{*}$ second \\
& Security check & Delay time & uniform $(3.0,6.0){ }^{*}$ second \\
& Gate & Delay time & exponential $(3.0){ }^{*}$ second \\
& Escalator & Speed & $0.65 \mathrm{~m} / \mathrm{s}$ \\
\hline
\end{tabular}

In the simulation model, the pedestrian speed is $1 \mathrm{~m} / \mathrm{s}$. The subway train serving this station consists of six vehicles, and each vehicle is fitted out with four train doors. The headway time and dwell time running by the current timetable are $150 \mathrm{~s}$ and $30 \mathrm{~s}$. Assume that there are no remaining passengers on the platform at the end of each alighting-boarding cycle. Total numbers of alighting passengers in each cycle during non-peak time and peak time are deterministic and equal to 160 and 510, respectively (In the Beijing subway, the peak time includes 7:00-9:00 a.m. and 6:00-8:00 p.m. and the rest of the operation time is non-peak time.). Passenger routing probabilities are estimated as the ratio of the passengers who arrived at an entrance and followed the specific route, by the total number of passengers who entered the system from this entrance. Other passenger flow data is described in Table 5.

Table 5. Passenger flow volume at each entrance.

\begin{tabular}{ccccc}
\hline Entrance & A & B & C & D \\
\hline Passenger flow volume during non-peak time (person $/ \mathrm{h})$ & 400 & 680 & 420 & 580 \\
Passenger flow volume during peak time (person/h) & 1200 & 1480 & 1160 & 1390 \\
\hline
\end{tabular}

Due to the randomness of AnyLogic simulation results, multiple simulations experiments are carried out and the average value of the results is adopted. During non-peak time, the real time of the departure process and the arrival process is $134 \mathrm{~s}$ and $92 \mathrm{~s}$, respectively. And the simulation time of the departure process and the arrival process is $130 \mathrm{~s}$ and $89 \mathrm{~s}$, respectively. During peak time, the real time of the departure process and the arrival process is $187 \mathrm{~s}$ and $155 \mathrm{~s}$, respectively. The simulation time of the departure process and the arrival process is $178 \mathrm{~s}$ and $148 \mathrm{~s}$, respectively. The errors between simulation results and actual data are small. Therefore, the accuracy of the simulation model is verified. 


\subsection{Sensitivity Analysis}

When other equipment is working properly, the impact of the number of failed gates on $T_{A}$ is shown in Figure 8. The two escalators at the station are both upward escalators, and the impact of the number of failed escalators on $T_{A}$ is shown in Figure 9.

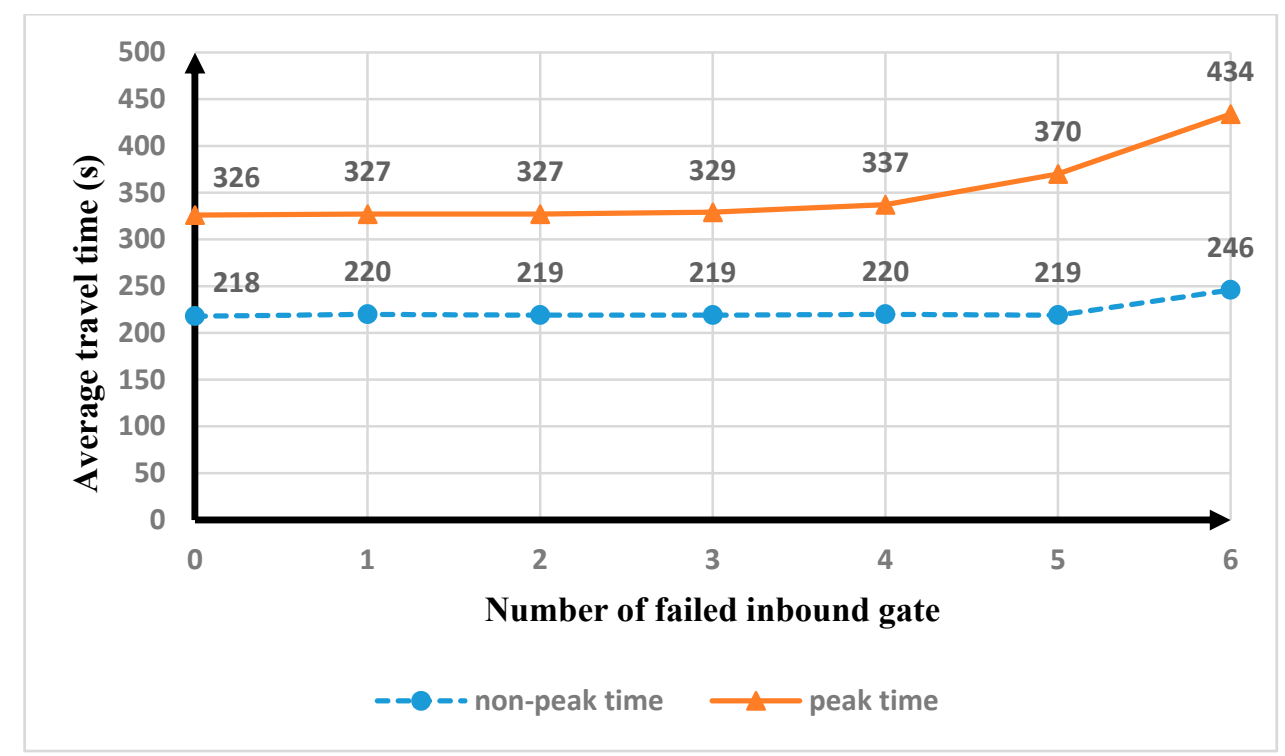

(a)

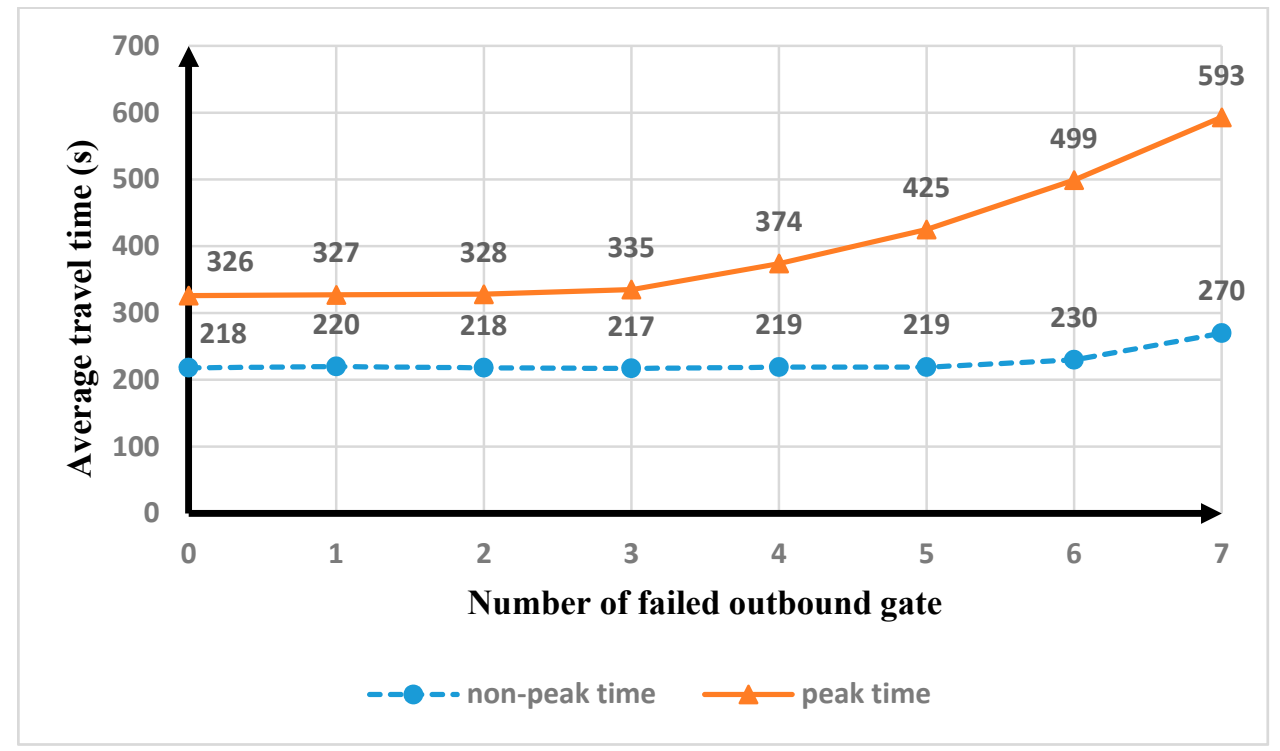

(b)

Figure 8. The impact of the number of failed gates on average travel time. (a) The impact of the number of failed inbound gates on average travel time; (b) The impact of the number of failed outbound gates on average travel time. 


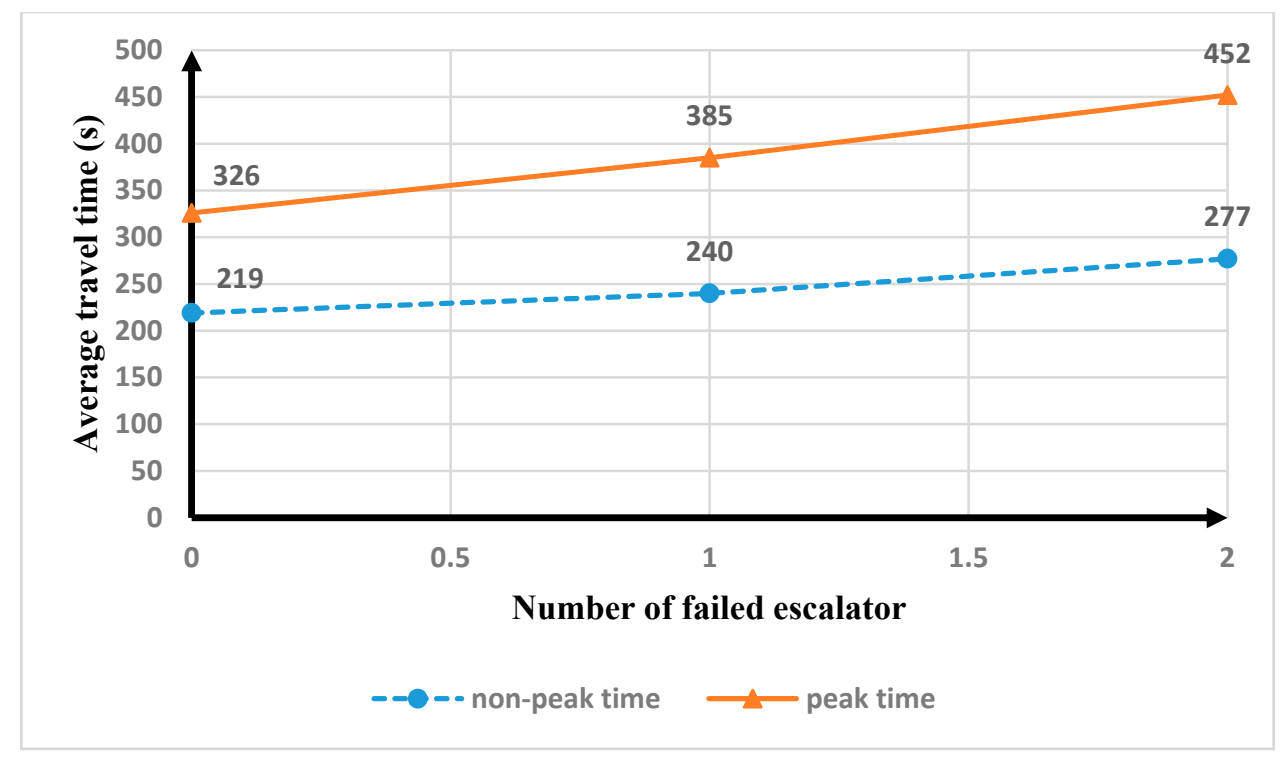

Figure 9. The impact of the number of failed escalators on average travel time.

As is shown in Figure 8, the impact of equipment failure on the DSSC during peak time is much more severe, therefore, the equipment that failed during peak time needs to be repaired in time. During peak time, when the number of failed inbound gates is less than 3 or the number of failed outbound gates is less than 2, the average travel time is almost unchanged. Since the gates are not the capacity bottleneck of the station, the travel time of passengers is not affected under the current passenger flow conditions. When the number of failed inbound gates is more than 3 or the number of failed outbound gates is more than 2, the average travel time increases rapidly and gates need to be repaired in time. As for the inbound gate, $\phi_{3}=0.9 \%$ and $\phi_{4}=3.3 \%$. As for the outbound gates, $\phi_{2}=0.6 \%$ and $\phi_{3}=2.7 \%$. When the sensitivity threshold $\beta$ is $1 \%$, the critical value of failed inbound gates is 3 and the critical value of failed outbound gates is 2 . In addition, the impact of outbound gate failure on the DSSC is much more severe. Therefore, the failed outbound gates deserve higher priority for repair. As is shown in Figure 9, the travel time increases significantly when the escalator fails, and the average travel time also becomes longer with the increase of the number of failed escalators. When the escalator fails, the route from the platform to the hall is reduced, and the passenger streamline is more conflicted on the stairs. It is necessary to prevent escalators from malfunctioning, especially during rush hour. During peak time, $\phi_{1}=18.1 \%$ and $\phi_{2}=38.7 \%$. Therefore, the maintenance priority of station equipment is as follows: the escalators have higher priority for repair than the gates, and the outbound gates have higher priority for repair than the inbound gates.

The station capacity model with equipment failure is used to analyze the impact of the reliability of the key equipment on the expected DSSC (also referred to as expected station capacity). When all inbound gates or outbound gates fail, the travel time is infinite in principle. However, in order to measure time and the unit time granularity of calculating station capacity is $1 \mathrm{~h}$, the travel time is assigned as $3600 \mathrm{~s}$ in the simulation study. When the reliability of the other equipment is 1 , the impact of the reliability of gate on the expected DSSC is shown in Figure 10. Similarly, the impact of the reliability of escalator on the expected DSSC is shown in Figure 11.

As shown in Figure 10, as the reliability of the gate increases, the expected average travel time gradually decreases and remains stable after the reliability reaches 0.7 . When the reliability of the gate is 0.6 , the sensitivity of the inbound gate and the outbound gate is $4.3 \%$ and $11.3 \%$, respectively. When the reliability of the gate is 0.7 , the sensitivity of the inbound gate and the outbound gate is $1.5 \%$ and $4.9 \%$, respectively. When the sensitivity threshold $\gamma$ is $10 \%$, the reliability of the gate needs to be maintained at 0.7 or higher in the current passenger flow conditions. Similarly, when the reliability of the escalator is 0.7 , the sensitivity of the escalator is $11.0 \%$, when the reliability of the escalator is 0.8 , 
the sensitivity of the escalator is $7.3 \%$. Hence, the reliability of the escalator needs to be maintained at 0.8 or higher. When key equipment does not reach the specified reliability, maintenance measures need to be taken to maintain station capacity.

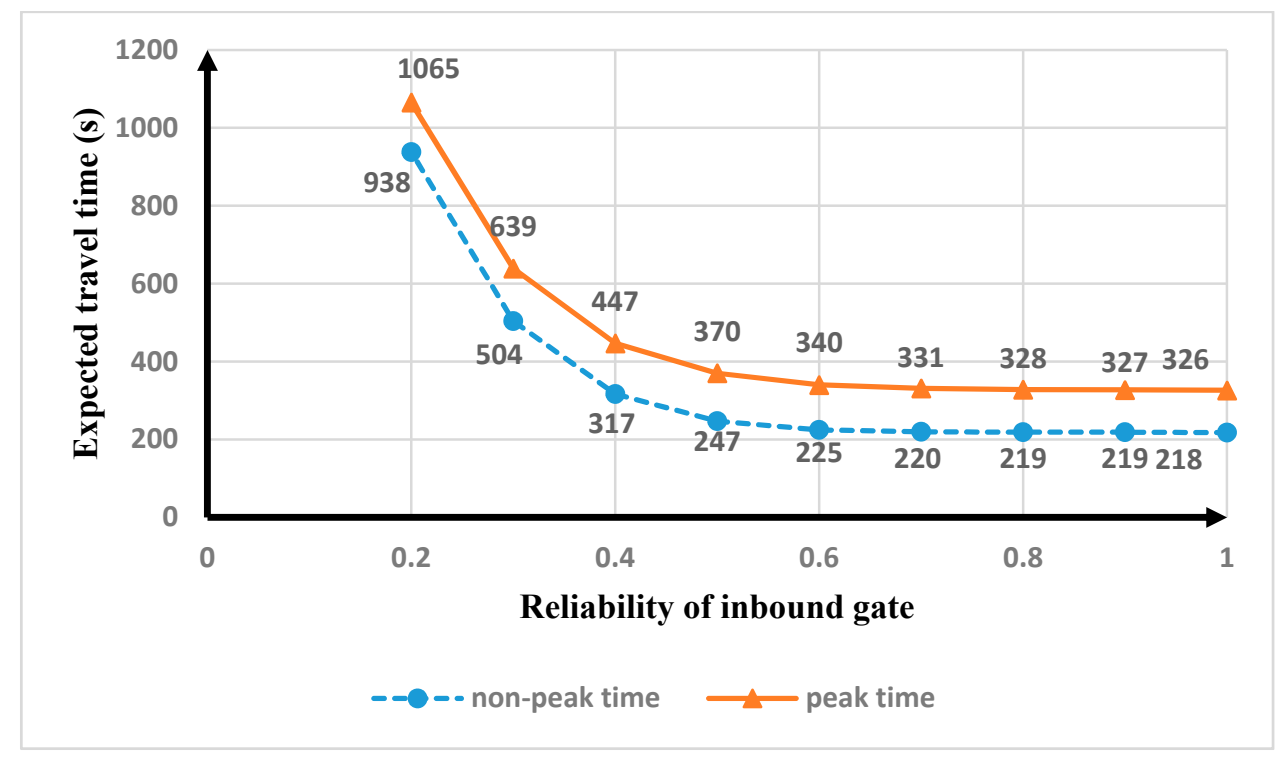

(a)

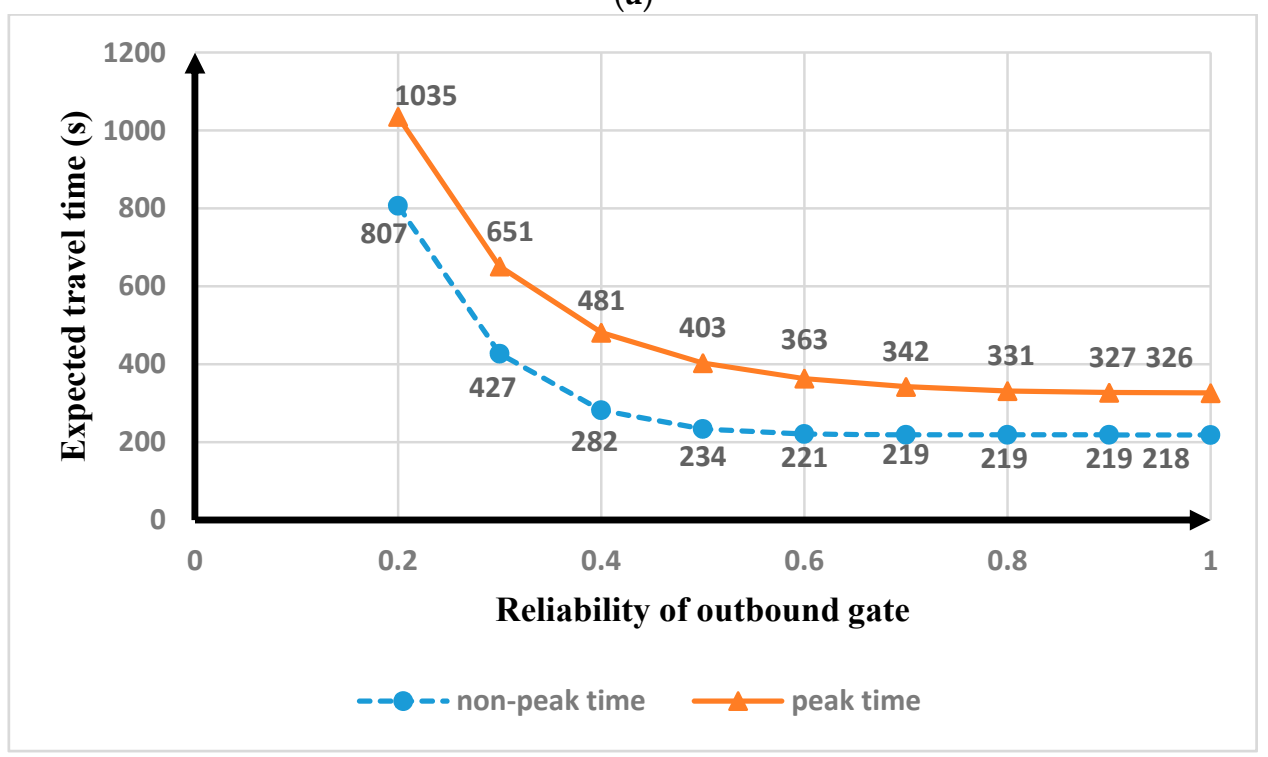

(b)

Figure 10. The impact of the reliability of the gate on expected travel time. (a) The impact of the reliability of the inbound gate on expected travel time; (b) The impact of the reliability of the outbound gate on expected travel time. 


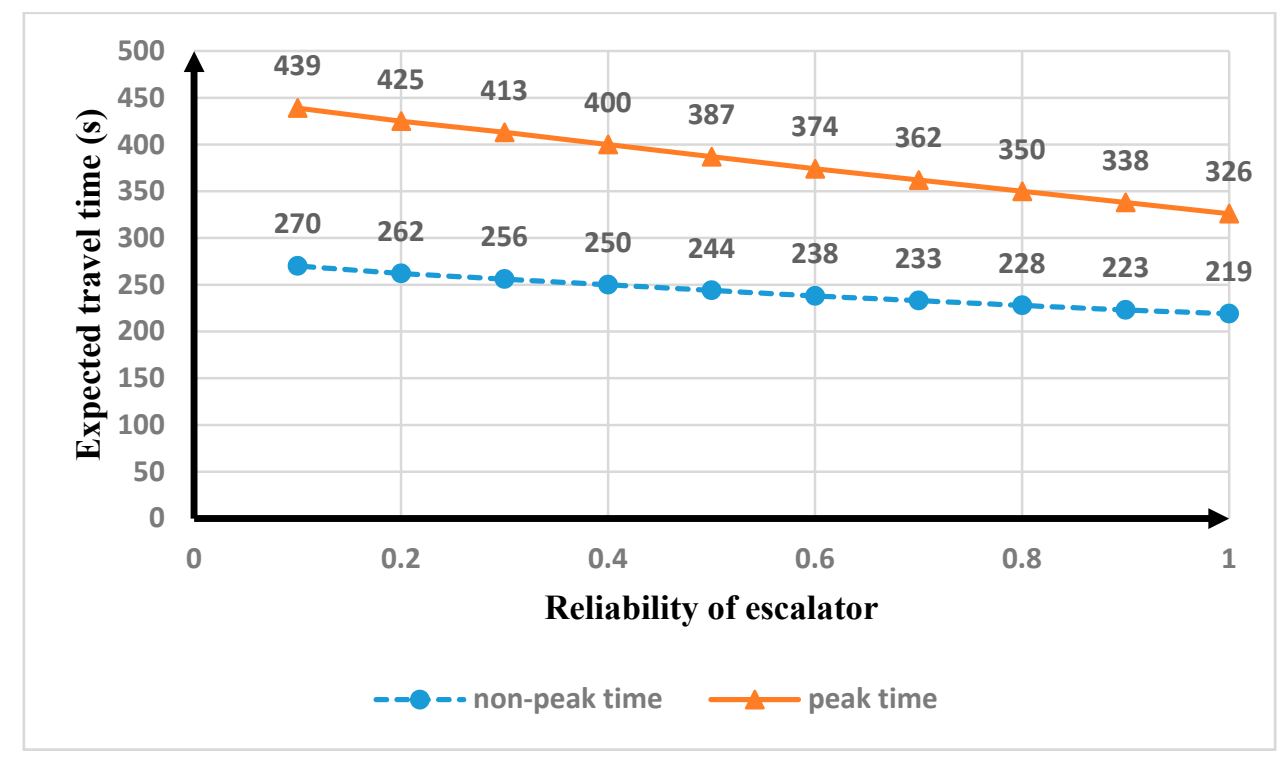

Figure 11. The impact of the reliability of the escalator on expected travel time.

\subsection{Reliability Modeling of the Key Equipment}

In this section, the reliability modeling issue is presented for the two kinds of key equipment: the gate and the escalator. The fault data are obtained from the maintenance records of the subway company. In this paper, the maximum likelihood estimation method (MLE) is selected to estimate parameters of fault distributions, and the Anderson-Darling statistic is used to select the optimal distribution.

\subsubsection{Reliability Modeling of the Gate}

The components of the gate include the box body, the blocking body, the machine core, the control module and the auxiliary module. The failure-prone components of the gate are mainly the machine core and control module. In the light of the maintenance records of the gate, the faults of the gate can be divided into two categories: software faults and hardware faults. The common software fault is the system crash which requires restart of the gate. The familiar hardware faults include poor contact of circuit, abnormal component parameters and component damage which requires replacement of components. Based on the statistical analysis of fault data, the software faults account for $43 \%$ and the hardware faults account for $57 \%$. The software and hardware fault distribution fitting are completed separately.

In this study, 51 samples of the software fault intervals of the gate are used. The average fault interval is 34 days, and the standard deviation is 40 . The result of goodness of fit test is shown in Figure 12. As shown in Figure 12, Weibull distribution fits the fault data best, and the Anderson-Darling statistic of Weibull distribution is the smallest. Therefore, the optimal fault distribution of software is Weibull distribution, and the shape parameter is 0.80 , the scale parameter is 30.05 . For the hardware fault of the gate, 63 samples of the fault interval are used. The average fault interval is 62 days, and the standard deviation is 82. The result of goodness of fit test is shown in Figure 13. As shown in Figure 13, Weibull distribution fits the fault data best, and the Anderson-Darling statistic of Weibull distribution is the smallest. Therefore, the optimal fault distribution of hardware is Weibull distribution, and the shape parameter is 0.843 , the scale parameter is 55.92. The reliability function of Weibull distribution is formulated as follows:

$$
R(k)=\exp \left[-\left(\frac{k}{\eta}\right)^{\beta}\right]
$$

where $k>0, \beta>0$ and $\eta>0, \beta$ is the shape parameter and $\eta$ is the scale parameter. 


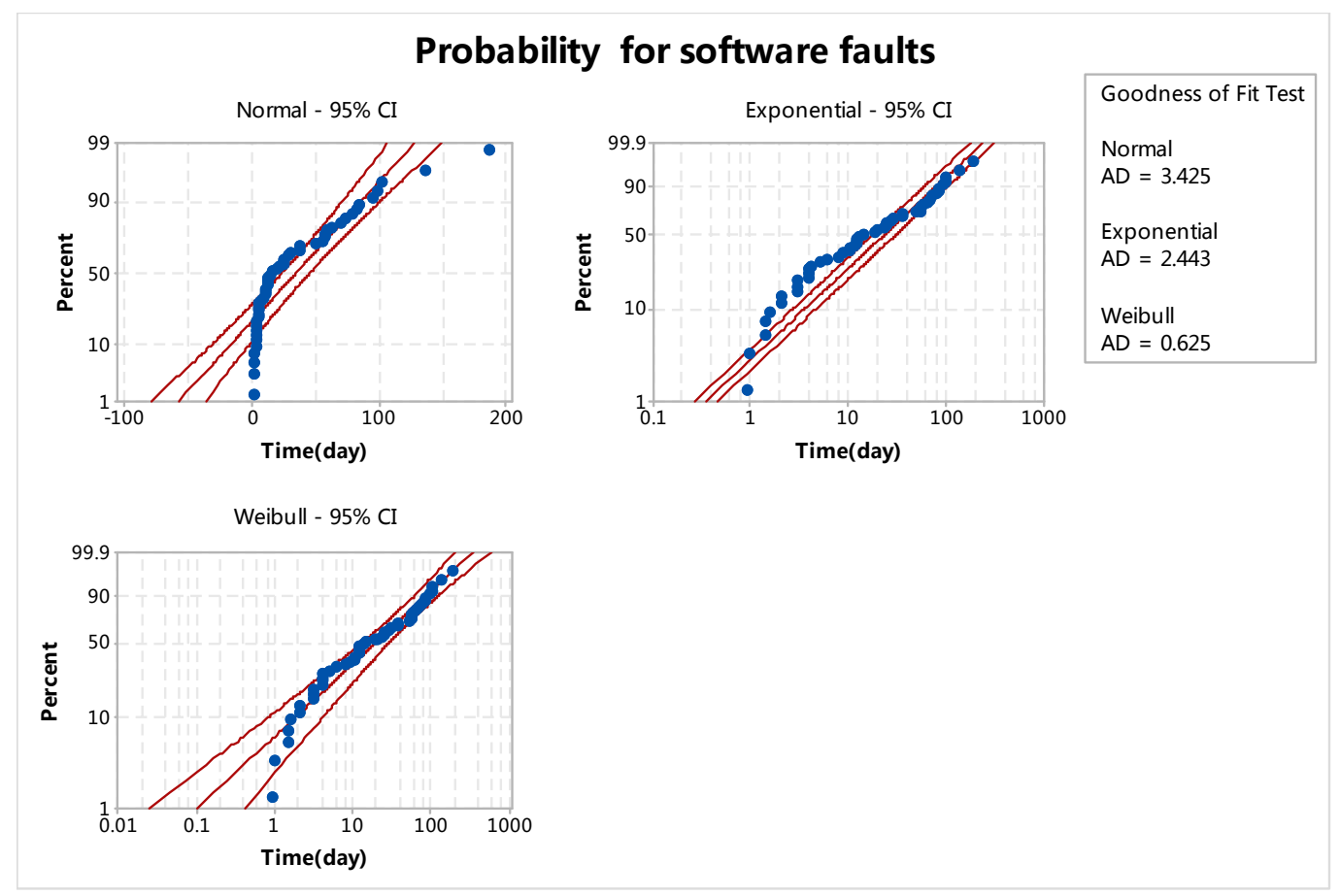

Figure 12. Goodness of fit test for software faults.

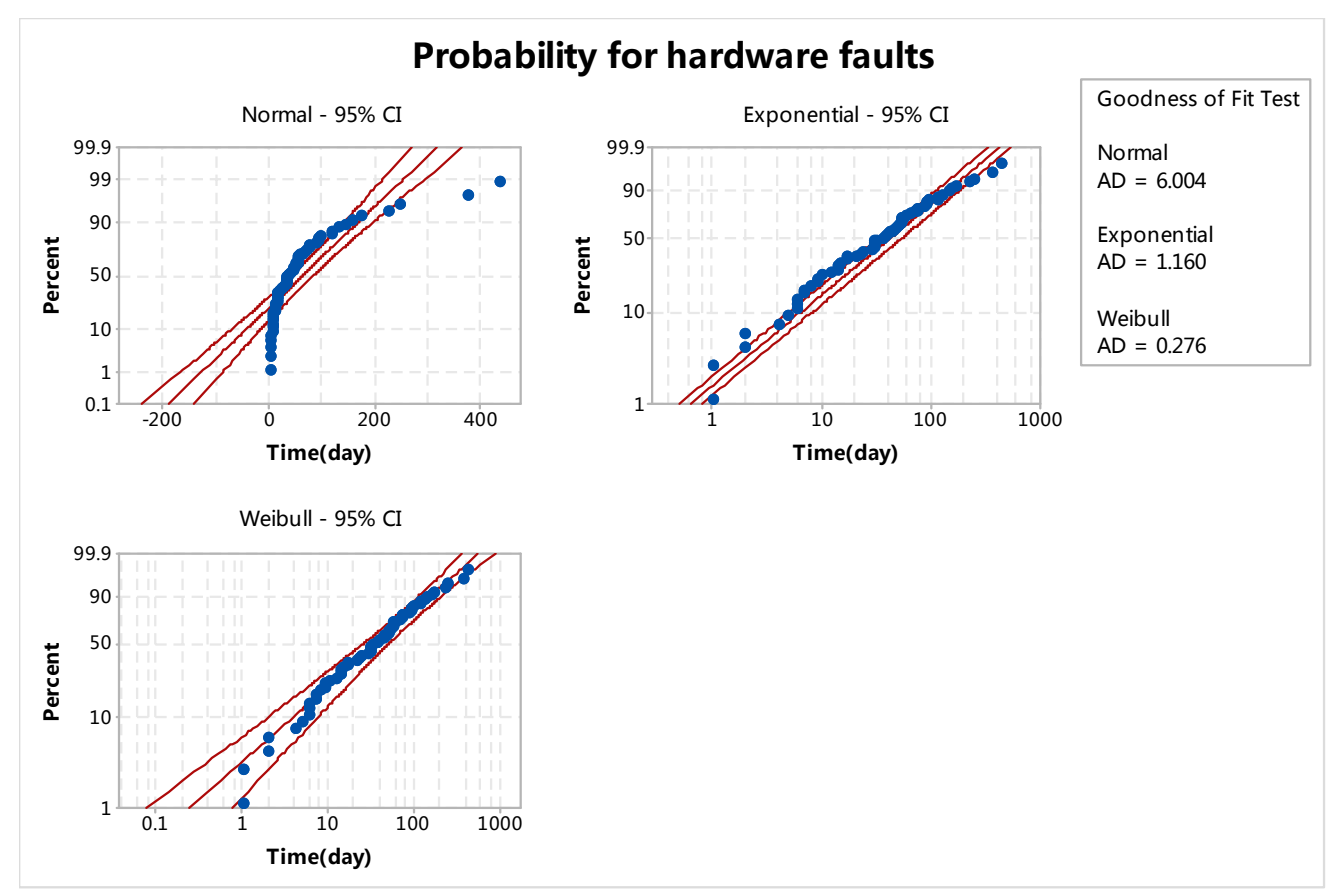

Figure 13. Goodness of fit test for hardware faults.

\subsubsection{Reliability Modeling of the Escalator}

The escalator is a large and complex form of transportation equipment with a mechanical structure as the main body. In terms of its functions, the escalator is divided into eight parts: support structure, escalator step system, guide rail system, handrail drive system, handrail device, safety protection device, electrical control system and automatic lubrication device. In light of the maintenance records of the escalator, the faults of the escalator are divided into two categories: intrinsic faults and faults caused by external environment factors, such as passenger interference and foreign objects intrusion. The intrinsic faults are divided into electrical system faults and mechanical system faults. Electrical 
system faults include control system fault, safety switch action, relay defective and etc., which need to reset control system and all kinds of switches. Mechanical system faults include handrail belt fault, motor bearing fault, drive chain fault and etc., which need to be adjusted or replaced. Based on the statistical analysis of fault data, the faults caused by external environment factors account for $34 \%$, which are not considered since they are uncontrollable, and the intrinsic faults account for $66 \%$, among which electrical system faults account for $37 \%$ and mechanical system faults account for $29 \%$. These two class of faults can be repaired and are considered in the maintenance schedule optimization. The electrical system and mechanical system fault distribution fitting are done separately.

For the electrical system faults of the escalator, 29 samples of the fault interval are used. The average fault interval is 94 days, and the standard deviation is 125 . The result of goodness of fit test is shown in Figure 14. As is shown in Figure 14, Weibull distribution fits the fault data best, and the Anderson-Darling statistic of Weibull distribution is the smallest. Therefore, the optimal fault distribution of electrical system is Weibull distribution, and the shape parameter is 0.91 , the scale parameter is 88.23 . For the mechanical system fault of the escalator, 28 samples of the fault interval are used. The average fault interval is 161 days, and the standard deviation is 212 . The result of the goodness of fit test is shown in Figure 15. As is shown in Figure 15, Weibull distribution fits the fault data best, and the Anderson-Darling statistic of Weibull distribution is the smallest. Therefore, the optimal fault distribution of the mechanical system is Weibull distribution, and the shape parameter is 0.923 , the scale parameter is 153.69 .

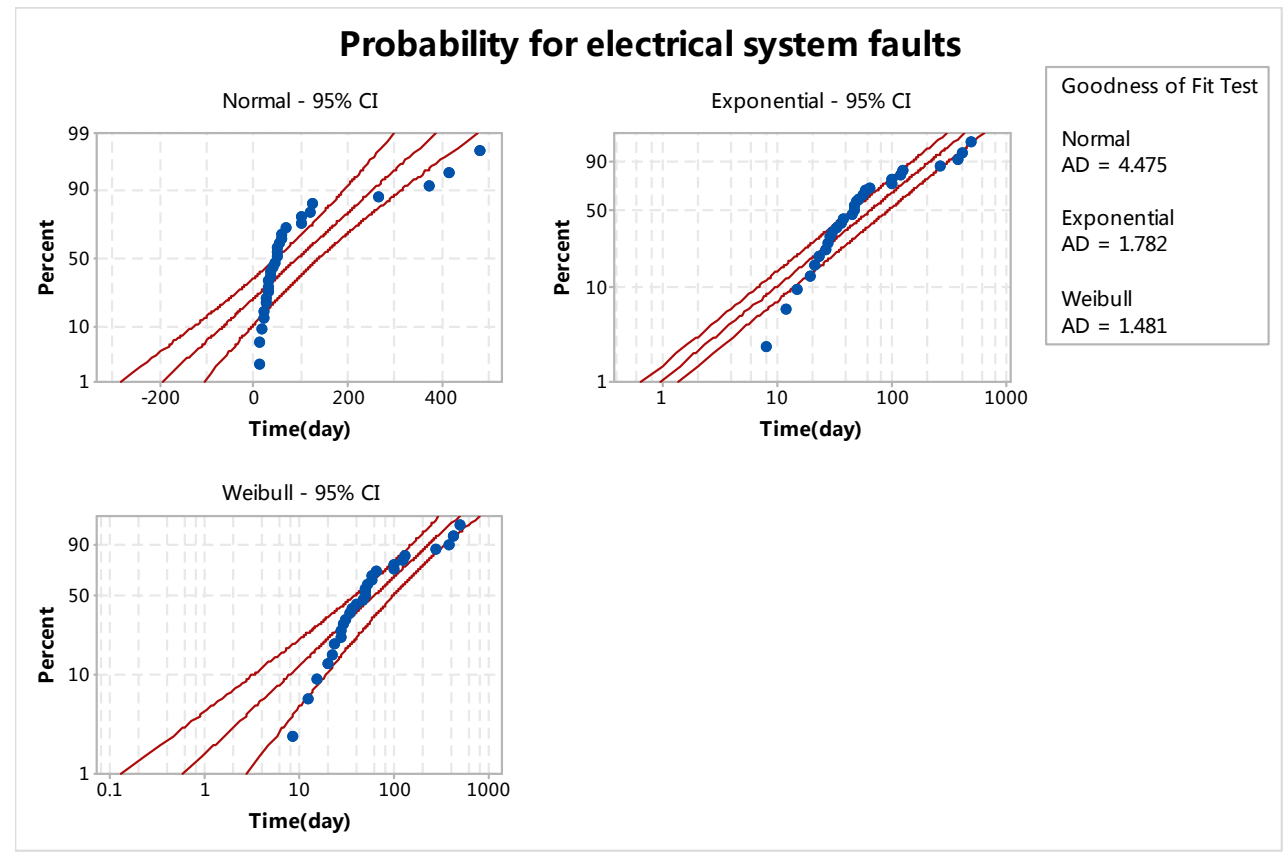

Figure 14. Goodness of fit test for electrical system faults. 


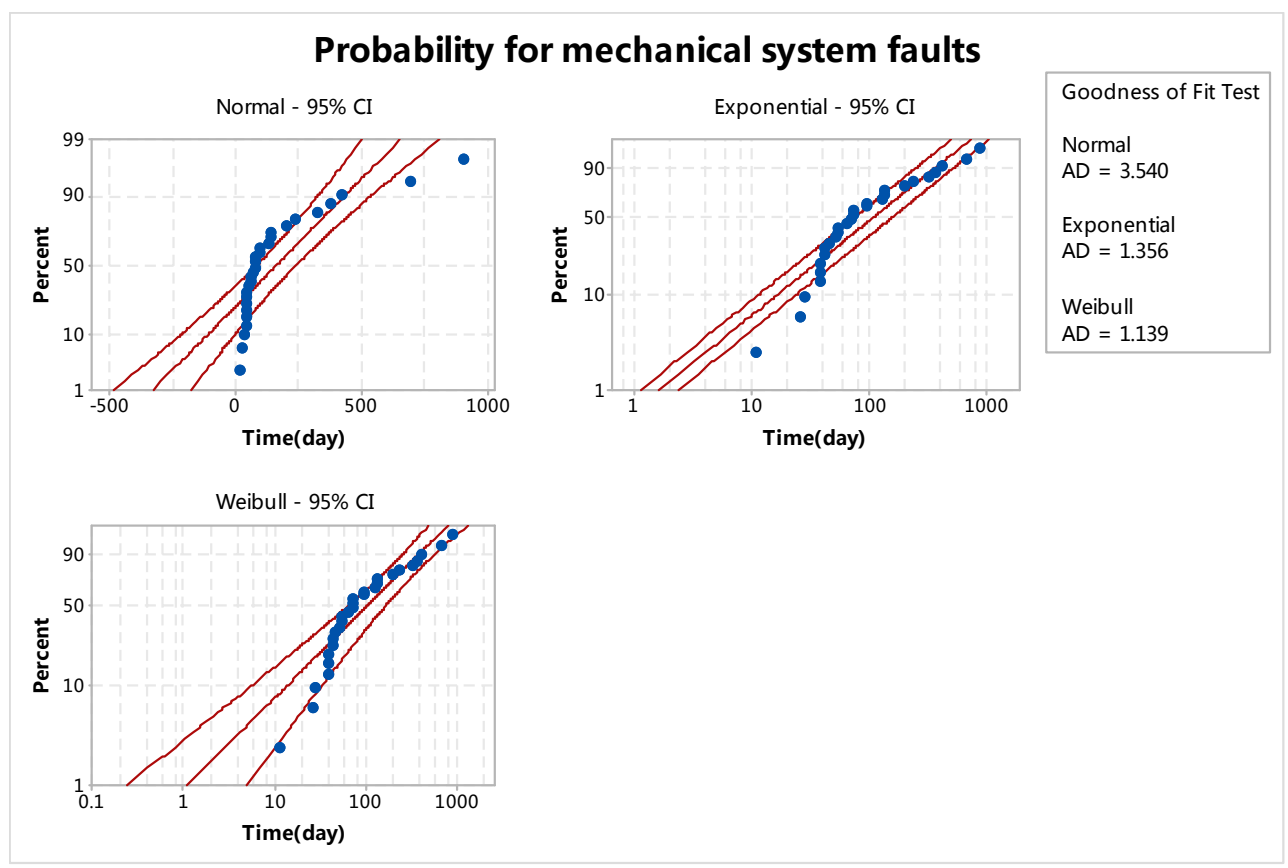

Figure 15. Goodness of fit test for mechanical system fault.

\subsection{Maintenance Schedule Optimization}

The optimal fault distribution of each components of the key equipment and the corresponding parameters are determined. In addition, the maintenance cost data is obtained from the maintenance account records. The maintenance cost of the gate software, $c_{p 1}$ is $100 \mathrm{RMB}$ and $c_{f 1}$ is $300 \mathrm{RMB}$. For the gate hardware, $c_{p 2}$ is $250 \mathrm{RMB}$ and $c_{f 2}$ is $1000 \mathrm{RMB}$. For the electrical system of the escalator, $c_{p 3}$ is $500 \mathrm{RMB}$ and $c_{f 3}$ is $1500 \mathrm{RMB}$. For the mechanical system of the escalator, $c_{p 4}$ is $1000 \mathrm{RMB}$ and $c_{f 4}$ is 3000 RMB.

The lower bound of equipment reliability is determined by sensitivity analysis based on the equipment reliability-expected station capacity curve. The lower bound of reliability of gate is 0.7 , and the lower bound of reliability of escalator is 0.8 . The relevant parameters of the equipment are taken into the maintenance schedule optimization model, and MATLAB is used to get a solution of the model. As a result, the optimal software maintenance schedule of the gate $T_{1}$ is 3 days, and the optimal hardware maintenance schedule of the gate $T_{2}$ is 8 days. The optimal maintenance schedule of the electrical system of the escalator $T_{3}$ is 7 days, and the optimal maintenance schedule of the mechanical system of the escalator $T_{4}$ is 16 days. Therefore, the optimal maintenance strategy of the gates is as follows: the maintenance schedule of software is 3 days, and the maintenance schedule of the gate is one week. The optimal maintenance strategy of the escalators is as follows: the maintenance schedule of the electrical system is one week, and the maintenance schedule of the escalator is half a month.

The current maintenance schedule of gates is half a year and the gates are repaired after failure in the majority of cases. The current maintenance schedule of escalators is 2 weeks. The equipment reliability between the current maintenance schedules and the optimal maintenance schedules are compared. The comparison result of gates is shown in Figure 16 and the comparison result of escalators is shown in Figure 17. As shown in Figure 16, the initial reliability of the gate is 1, and the reliability decreases as the operation time goes by. After the maintenance for software system of gates on the third day, the reliability of the gate is restored to 0.9 on the fourth day. The reliability of gates is decreased further as operation time goes on and decreases to 0.7 at the seventh day. After the maintenance for the entire gate on the seventh day, the reliability of the gate is restored to 1 again on the eighth day. The change of the escalator reliability before and after maintenance is shown in Figure 7. 


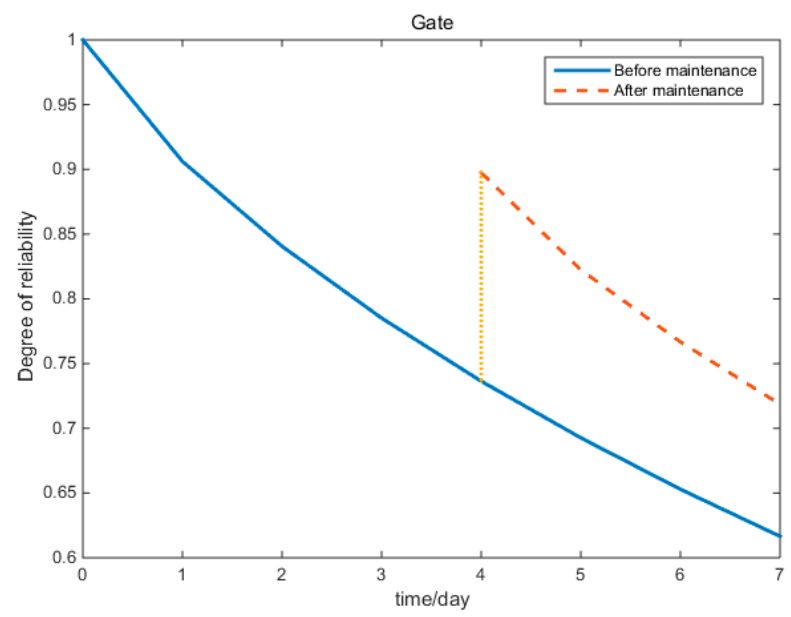

Figure 16. The reliability change due to the maintenance of gates.

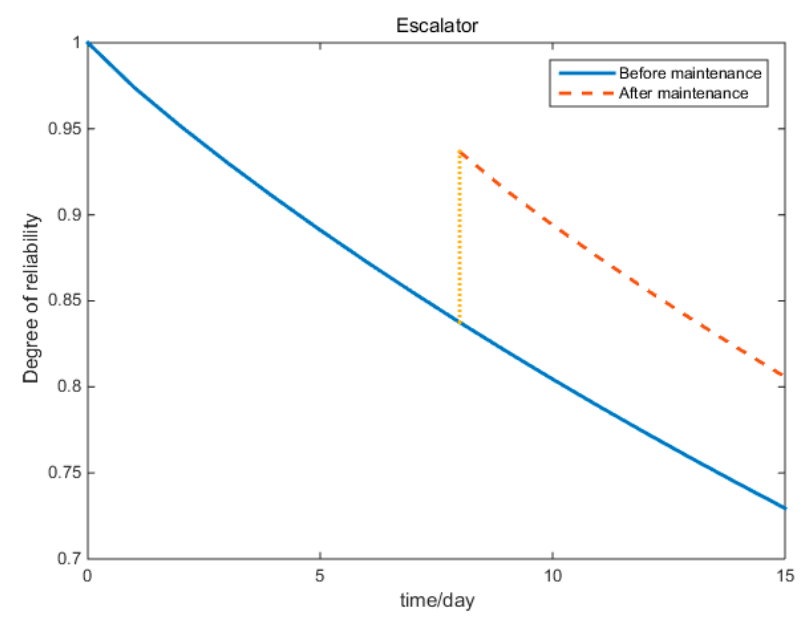

Figure 17. The reliability change due to the maintenance of escalators.

Figure 18 shows the variation of the expected DSSC during rush hours over operation time after maintenance schedule optimization, which is evaluated with expected travel time of passengers. In the beginning, the reliability of gates and escalators is 1 , and the expected travel time is $326 \mathrm{~s}$. As operation time goes by, the reliability of key equipment decreases and the expected travel time increases. After the maintenance for software system of gates on the third day, the reliability of gates is restored to 0.9 , and the expected travel time reduces to $337 \mathrm{~s}$. The expected travel time increases again with operation time. After the maintenance for gates and electrical system of escalators on the seventh day, the reliability of gates and escalators is restored to 1 and 0.95 , respectively, and the expected travel time decreases again. After the maintenance for software system of the gates on the eleventh day, the expected travel time remains almost unchanged. Finally, the expected DSSC is restored to the initial level after equipment maintenance every half a month. Before the maintenance schedule optimization, the failure probability of key equipment increases and the expected DSSC declines without timely maintenance. From the above analysis, the reliability of key equipment is maintained with the optimal maintenance schedules and the station capacity is remained in a certain level by maintaining the reliability of key equipment. 


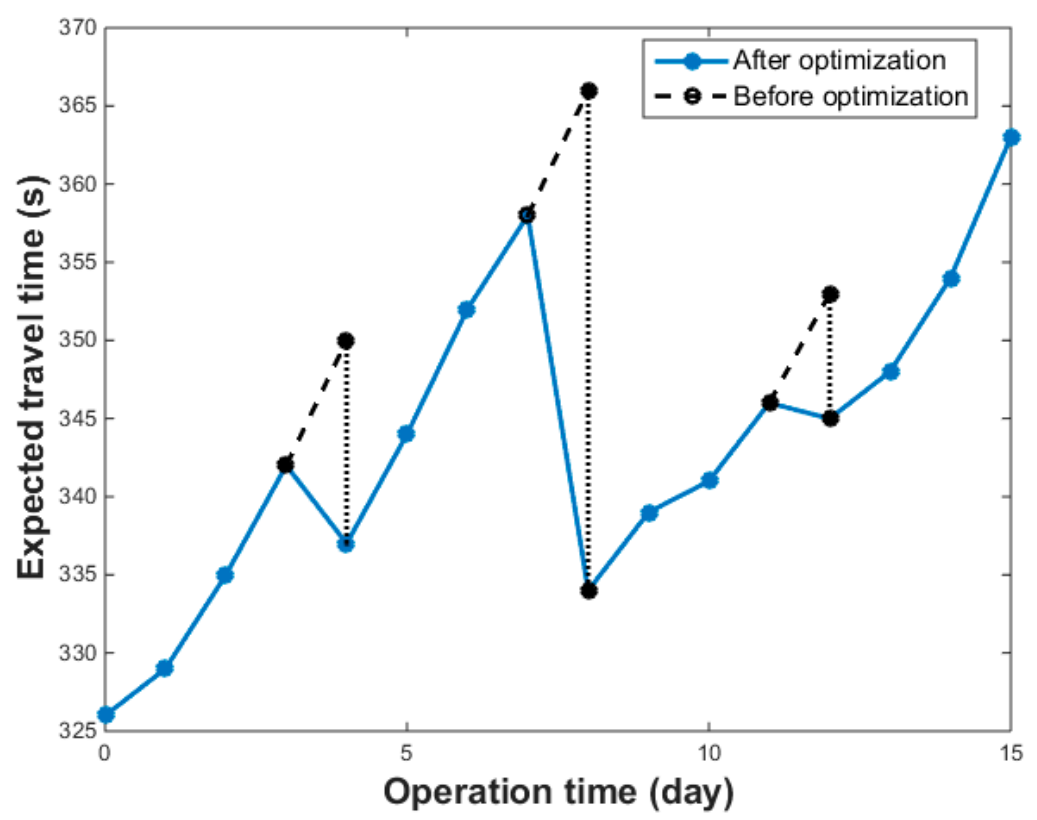

Figure 18. Expected travel time change with incorporating reliability and maintenance levels.

\section{Conclusions}

In this paper, the dynamical subway station capacity (DSSC) is defined according to the gathering and scattering process of the subway station and the travel time of passengers is taken as the DSSC indicator. In consideration of key equipment failure, the expected DSSC model is established based on the idea of binomial distribution and mathematical expectation. After that, the lower bound of equipment reliability is determined by sensitivity analysis based on the equipment reliability-expected station capacity curve. Finally, the maintenance schedule optimization model is established to maintain DSSC with the lowest cost under the constraint of equipment reliability, and a solution to the maintenance optimization model is also proposed. The simulation results demonstrate that the station capacity can be maintained in a level range by using the proposed method of maintenance schedule optimization. This provides a useful alternative for subway operation companies. This is the first contribution which aims at maintaining the station capacity while considering key equipment failure by optimizing the maintenance schedule of the key equipment.

There are still some technical issues need to be considered. For example, a more general expected capacity calculation framework may be considered, in which passenger route assignment aims to achieve the maximum capacity of the subway station subject to equipment failure. In addition, the age regression factor may be considered in maintenance schedule optimization, since not all equipment can be restored to a completely new state after maintenance. These areas will be our work in the future.

Author Contributions: S.L. collected and analyzed the data, made charts and diagrams, conceived and performed the experiments and wrote the paper; X.W. conceived the structure, modified the manuscript and provided guidance; Z.Z. provided the idea of station capacity model and searched the literature; L.J. modified the manuscript. J.Y. provided the method to analyze the fault data of station equipment.

Funding: This research is supported by National Key R\&D Program of China (Contract No. 2017YFB1201203).

Acknowledgments: This research is also supported by State Key Laboratory of Rail Traffic Control and Safety (Contract No. RCS2019ZT004) and China Postdoctoral Science Foundation (2018M641174).

Conflicts of Interest: The authors declare no conflict of interest. 


\section{References}

1. Washington Metropolitan Area Transit. Metrorail Station Access \& Capacity Study; WMATA: Washington, DC, USA, 2008.

2. Hu, Q.-M. Passenger Carrying Capacity Evaluation and Simulation of Rail Transit Station. Ph.D. Dissertation, Beijing Jiaotong University, Beijing, China, 2011. (In Chinese)

3. Cruz, F.R.B.; MacGregor Smith, J.; Medeiros, R.O. An M/G/C/C state-dependent network simulation model. Comput. Oper. Res. 2005, 32, 919-941. [CrossRef]

4. Cruz, F.R.B.; MacGregor Smith, J. Approximate analysis of M/G/c/c state-dependent queueing networks. Comput. Oper. Res. 2007, 34, 2332-2344. [CrossRef]

5. Yalcınkaya, Ö.; Mirac Bayhan, G. Modelling and optimization of average travel time for a metro line by simulation and response surface methodology. Eur. J. Oper. Res. 2009, 196, 225-233. [CrossRef]

6. Fernández, R. Modelling public transport stops by microscopic simulation. Transp. Res. Part C Emerg. Technol. 2010, 18, 856-868. [CrossRef]

7. Chen, S.-K.; Liu, S.; Xiao, X.; Hong, J.; Mao, B.-H. M/G/c/c-based model of passenger evacuation capacity of stairs and corridors in metro stations. J. China Railw. Soc. 2012, 34, 7-12. (In Chinese)

8. Xu, X.-Y.; Liu, J.; Li, H.-Y.; Jiang, X. Calculation of Gathering and Distributing Capacity of subway station by simulation and response surface methodology. J. China Railw. Soc. 2013, 35, 8-18. (In Chinese)

9. Xu, X.-Y.; Liu, J.; Li, H.-Y.; Hu, J.-Q. Analysis of subway station capacity with the use of queueing theory. Transp. Res. Part C Emerg. Technol. 2014, 38, 28-43. [CrossRef]

10. Teknomo, K. Application of microscopic pedestrian simulation model. Transp. Res. Part F Traffic Psychol. Behav. 2006, 9, 15-27. [CrossRef]

11. Kaakai, F.; Hayat, S.; El Moudni, A. A hybrid Petri nets-based simulation model for evaluating the design of railway transit stations. Simul. Model. Pract. Theory 2007, 15, 935-969. [CrossRef]

12. Asano, M.; Iryo, T.; Kuwahara, M. Microscopic pedestrian simulation model combined with a tactical model for route choice behaviour. Transp. Res. Part C Emerg. Technol. 2010, 18, 842-855. [CrossRef]

13. Li, H.-X.; Li, H.-Y.; Fan, X.; Xu, X.-Y. Anylogic-based simulation analysis and evaluation of subway stations assemble capacity. Railw. Comput. Appl. 2012, 21, 48-50. (In Chinese)

14. Zhao, L.-M.; Zheng, Y.; Xie, J.-X. Simulation of Urban Transit station based on AnyLogic. Railw. Comput. Appl. 2016, 3, 62-66. (In Chinese)

15. Alexandre, G. Simulation on the Serviceability and Emergency Evacuation of a Subway Station System by AnyLogic. Master's Thesis, Harbin Institute of Technology, Harbin, China, 2018.

16. Grudén, M.; Westman, A.; Platbardis, J.; Hallbjorner, P.; Rydberg, A. Reliability experiments for wireless sensor networks in train environment. In Proceedings of the European Wireless Technology Conference, Rome, Italy, 28-29 September 2009; pp. 37-40.

17. Lu, Y.; Zeng, J.; Wu, P.; Yang, F.; Guan, Q. Reliability and parametric sensitivity analysis of railway vehicle bogie frame based on Monte-Carlo numerical simulation. In High Performance Computing and Applications; Springer: Berlin/Heidelberg, Germany, 2010; pp. 280-287.

18. Wang, S.-F.; Zhao, H.-L. Reliability Design and Research on Passenger Car Bogie System. Roll. Stock 2007, 45, 1-3. (In Chinese)

19. Zheng, J.-C. Reasearch on the Reliability of Bogie System of High-Speed Train. Master's Thesis, Beijing Jiaotong University, Beijing, China, 2016. (In Chinese)

20. Mao, Y.-X. Planning and Implementation of RCM System for Nanjing Metro Facilities and Equipment. Master's Thesis, Nanjing University of Science and Technology, Nanjing, China, 2008. (In Chinese)

21. Chen, C.-H.; Xu, Y.-N.; Fu, X.-L. Optimized Model of Maintenance Cycle for Key Metro Traffic Operation Equipment and Its Application. Urban Rapid Rail Transit 2011, 24, 42-45. (In Chinese)

22. Lu, L.-B.; Gao, Q.; Li, Y.-W.; Ge, Y.; Zhang, H. Maintenance modeling and optimization of equipment based on Monte Carlo simulation. Comput. Digit. Eng. 2011, 39, 47-49. (In Chinese) 
23. Yu, D.-D. Research on the Optimization and Methods of Urban Rail Transit Vehicle Overhaul Model Based on Reliability. Master's Thesis, Nanjing University of Science and Technology, Nanjing, China, 2017. (In Chinese)

24. Chen, A.; Yang, H.; Lo, H.K.; Tang, W.H. Capacity reliability of a road network: An assessment methodology and numerical results. Transp. Res. Part B Methodol. 2002, 36, 225-252. [CrossRef] 